\title{
Philonsorbonne
}

12 | 2018

Année 2017-2018

\section{La naissance du sujet chez Louis Lavelle et Emmanuel Lévinas}

Sophie GALABRU

\section{OpenEdition}

1 Journals

Édition électronique

URL : https://journals.openedition.org/philonsorbonne/962

DOI : 10.4000/philonsorbonne.962

ISSN : $2270-7336$

Éditeur

Publications de la Sorbonne

Édition imprimée

Date de publication : 1 janvier 2018

Pagination : 45-59

ISSN : 1255-183X

\section{Référence électronique}

Sophie GALABRU, «La naissance du sujet chez Louis Lavelle et Emmanuel Lévinas », Philonsorbonne [En ligne], 12 | 2018, mis en ligne le 03 mars 2018, consulté le 09 juin 2021. URL : http:// journals.openedition.org/philonsorbonne/962 ; DOI : https://doi.org/10.4000/philonsorbonne.962

(c) Tous droits réservés 


\title{
La naissance du sujet chez Louis Lavelle et Emmanuel Lévinas
}

\author{
Sophie GALABRU
}

\section{Introduction}

Dès les années 1930, Emmanuel Lévinas revenait sur le privilège accordé à l'être par la pensée occidentale, pour ne jamais cesser, dans les œuvres suivantes, d'insister sur son caractère hégémonique étouffant selon lui la possibilité d'une autre pensée qu'il édifiera lui-même comme pensée de l'Autre. Ses premiers écrits manifestent la volonté de souligner l'omniprésence de l'être sur l'étant, remarquant dans De l'évasion (1935), le besoin de l'étant - manifestation individualisée de l'être - de prendre ses distances avec l'être même dont il est issu. Lévinas cherche alors à penser l'être non comme substantif mais comme verbe ou comme fait que De l'existence à l'existant (1947) et Totalité et Infini (1961) décrivent comme une présence impersonnelle, atemporelle, sous-jacente à tous les phénomènes et que l'individu doit assumer. En même temps que l'étant porte l'être, dont la présence est à vrai dire irrémissible, il se doit aussi de s'en dégager afin de l'investir de façon personnelle ; opération réalisée par la temporalisation du sujet. Cette attention prêtée à l'existence comme totalité soutenant l'existant ainsi que le rôle dévolu au présent dans la formation du sujet font écho aux écrits d'un grand métaphysicien français du $\mathrm{XX}^{\mathrm{e}}$ siècle, professeur et membre du Collège de France, Louis Lavelle, avec lequel Lévinas entretient un rapport certain.

L'évidence de ce rapport s'atteste dans la recension que Lévinas consacre à l'ouvrage de Lavelle intitulé La Présence totale dans les Recherches philosophiques de l'année 1934-1935. Lévinas commence d'ailleurs cet article ${ }^{1}$ en inscrivant La Présence totale dans la continuité

1. E. Lévinas, «Recension de L. Lavelle, La Présence totale », Recherches Philosophiques, IV, 1934/35, p. 392-395. 
d'un autre ouvrage de Lavelle paru en 1928 et dont il reprend les éléments essentiels ; De l'Être, qualifié d' «ouvrage capital »" puisque ce dernier inaugure en effet le système métaphysique de La Dialectique de l'éternel présent, entreprise d'envergure, comprenant trois autres volumes: De l'Acte (1937), Du Temps et de l'Éternité (1945) et De l'Âme humaine (1951) ainsi qu'un dernier De la Sagesse, jamais paru en raison de la disparition du philosophe. Lévinas ayant lu ces deux ouvrages, c'est à partir de ceux-ci que nous appuierons notre analyse. Nous verrons que Lavelle s'engage à redonner à l'être, entendu comme Tout et comme acte, ce qui lui revient de droit ; combattant d'un côté le subjectivisme sous sa forme idéaliste - faisant du sujet de la pensée la source du réel -, d'un autre côté le réalisme - pour lequel le sujet et la pensée ne sont que des épiphénomènes du réel. Par-delà cette opposition dialectique, Lavelle veut revenir au tout du réel, à cette présence totale sur fond de laquelle se constitue le sujet et dont le philosophe veut restituer la genèse, le replaçant dans sa véritable relation avec l'être. Il nous faut revenir sur cette pensée de l'être et du temps dont l'articulation promet la naissance du sujet. De cette manière, nous pourrons percevoir l'importance de l'héritage lavellien dans la pensée de Lévinas, ainsi que les limites que ce dernier perçoit et qui lui permettront de déterminer sa propre orientation philosophique.

\section{L’être conçu comme totalité et activité}

L'intérêt que Lévinas manifeste pour les ouvrages de Lavelle s'explique par ce souci partagé pour la question de l'être; question suscitée par les effets de la situation historique des années 1930 sur les deux penseurs. Lévinas voit en effet dans la réflexion lavellienne de l'être la manifestation d'une expérience tragique de la guerre qu'il connaît lui-même ${ }^{3}$ :

La guerre, les sombres pressentiments qui l'ont précédée et la crise qui l'a suivie rendirent à l'homme le sentiment d'une existence que la souveraine et impassible raison n'avait su ni épuiser, ni satisfaire ${ }^{4}$.

Ce que l'époque, par ses sombres évènements, a su révéler à la conscience humaine, c'est ce qui n'a en réalité jamais cessé de le préoccuper : l'existence et sa détermination temporelle. La souffrance et la mort sonnent l'urgence de revenir au fait d'exister dont l'omniprésence est

\section{Ibid., p. 392.}

3. Dans un entretien de 1987 avec F. Poirié, Lévinas déclare à propos de son article $D e$ l'évasion: "dans le texte originel, écrit, en 1935, on peut distinguer les angoisses de la guerre qui approchait et toute "la fatigue d'être", l'état d'âme de cette période ». Entretiens Emmanuel Lévinas/François Poirié, in François Poirié, Emmanuel Lévinas, Lyon, La Manufacture, 1987, p. 83.

4. E. Lévinas, « Recension de L. Lavelle, La Présence totale », op. cit., p. 392. 
toujours invisible pour l'homme insouciant. C'est ainsi que s'explique pour Lévinas la régénération de l'ontologie. Peu importe les interrogations sur la nature de ce qui est, sur l'existence du monde extérieur, il s'agit d'en revenir à ce qui ne nous quitte jamais sans que pourtant nous n'y prêtions attention : le fait d'être. Il y a donc une inanité de l'idéalisme comme du réalisme, mais aussi du scepticisme et de toutes ces formes spécifiques de la pensée philosophique qui, dans sa profondeur «se tient en dehors des discussions classiques sur l'existence ou la non-existence du monde extérieur $»^{5}$ dès lors qu'il s'agit d'en revenir à ce fait primordial. Ce dépassement de l'idéalisme et du réalisme n'échappe pas non plus à Lavelle pour qui ces positions représentent deux faces de la même vérité et dont l'exclusivité serait, quant à elle, une erreur. D'une part en effet, l'être ne peut être fondé par le sujet puisqu'il le soutient et le précède. D'autre part, l'être est irréductible au donné concret qu'il produit par un acte permanent par lequel le monde se fait et que la pensée imite. Ce que partagent nos deux auteurs, c'est donc ce fait de la présence de l'être ou plutôt cette idée que l'être est la présence, en deçà de ce qui se présente à soi - la conscience - ou aux autres, et ce, par-delà les débats infinis sur la nature de cette présence. C'est cette même intuition philosophique qui conduit à une même opposition au subjectivisme, poussant d'une part Lévinas à dire que « la notion de sujet ne suffit pas pour rendre compte de l'être $»^{6}$ et Lavelle à affirmer que :

le propre de la pensée n'est pas, comme on le croit, de nous séparer du monde, mais de nous y établir, qu'au lieu de nous resserrer sur nous-même, elle nous découvre l'immensité du réel dont nous ne sommes qu'une parcelle, mais qui est soutenue et non point écrasée par le Tout où elle est appelée à vivre?

Cette découverte de notre inclusion dans l'être, conduira chez l'un et l'autre à la description d'une dramaturgie de l'existence : non seulement je ne peux m'évader de cette présence ainsi que le diront Lavelle dans son Introduction à De l'être et Lévinas dans De l'évasion ${ }^{8}$, mais en outre, je suis astreint à assumer cette présence plus vaste que la mienne. Il y a là une preuve d'humilité de la part de la pensée qui peut toutefois confiner à l'assujettissement si l'on ne prend pas la mesure de l'appel à investir personnellement cette présence. Si pour Lavelle l'être ne dispose pas du

\footnotetext{
5. Ibid., p. 393.

6. Ibid.
}

7. L. Lavelle, La Présence totale, Paris, Fernand Aubier, aux Éditions Montaigne, $2^{\text {ème }}$ édition, 1934, Collection «Philosophie de l'esprit », p. 8.

8. «(..) la découverte de cette présence toujours actuelle dont je ne réussis jamais à m'évader », Id. De l'Être, Paris, Fernand Aubier, aux Éditions Montaigne, 1947, Collection «Philosophie de l'esprit », p. 9.

9. L'ouvrage entier traite du besoin de s'évader de l'être, et montre par l'impossibilité même où le moi se trouve d'y parvenir la forme dramatique de l'ontologie. Nous noterons que l'article parait dans Les Recherches philosophiques, l'année suivant sa recension de Présence totale. 
pouvoir de choisir puisqu'il est tout, il n'en impose pas moins de choisir notre façon d'être. C'est pourtant cette impossibilité de sortir de l'être, fondée chez Lavelle dans une philosophie de la participation à son principe interne, chez Lévinas sur notre inclusion inévitable en lui par notre naissance ${ }^{10}$, qui motive chez ce dernier le besoin d'évasion voire la révolte de l'individu envers cette présence. Cette possibilité du refus n'est pas énoncée chez Lavelle pour qui la découverte de l'être appelle, soit le consentement actif à l'être par lequel le moi s'affirme et imite l'activité créatrice de l'être, soit l'abandon passif par lequel le moi s'oublie et se laisse agir ${ }^{11}$. C'est pourtant la négation de cette inclusion à cette présence totale voire totalitaire ${ }^{12}$ qui caractérise le sujet lévinassien. Si Lévinas insiste donc sur la souffrance métaphysique induite par le fait irrémissible de la présence, Lavelle, lui, réduit cette souffrance à une possibilité psychique d'un individu mal réalisé ou dont l'ambition déçue peut pousser à vouloir en finir avec sa vie :

cette vie qui nous a été imposée, qui ne nous satisfait pas, et dont nous pouvons à chaque instant nous délivrer en la détruisant, est la condition d'un choix par lequel tout individu est appelé à accomplir un acte spirituel d'acceptation ou de renoncement ${ }^{13}$.

Le passage d'un niveau métaphysique à un niveau existentiel voire psychologique se remarque dans l'emploi du terme de vie ${ }^{14}$ quand l'auteur ne cessait de parler de l'être. Lorsque Lavelle ne psychologise pas ce refus, il le rend impossible d'un point de vue logique, manquant vraisemblablement sa teneur métaphysique: «Je ne m'évade jamais de l'être; et il est contradictoire que je puisse le nier, puisque je pose, en le niant, mon être qui le nie $»^{15}$. Lévinas, lui, prendra toute la mesure du rapport à l'être pour un

10. «L'entrée dans le monde est l'entrée dans l'être - dans ce fait qu'il y a - naissance Naissance de tous les instants ». E. Lévinas, Carnets de captivité, in Euvres I, Paris/Caen, Grasset/Imec, 2009, p. 60.

11. L'être en effet « rend possibles tous les choix chez un sujet qui, participant à sa nature, peut s'attacher, par un consentement qui fonde sa personne même, au principe intérieur qui l'anime et le fait être, ou bien s'abandonner à la nécessité (...) ». L. Lavelle, op. cit., p. 48.

12. «La paix des empires sortis de la guerre repose sur la guerre. Elle ne rend pas aux êtres aliénés leur identité perdue. Il y faut une relation originelle et originale avec l'être » écrit Lévinas dans Totalité et Infini, ouvrage dans lequel la guerre exprime une face de l'être et peut-être l'être même en tant que totalité qui contient et absout en elle les individus. En effet, comme il l'écrit : "La face de l'être qui se montre dans la guerre, se fixe dans le concept de totalité qui domine la philosophie occidentale. Les individus s'y réduisent à des porteurs de forces qui les commandent à leur insu. Les individus empruntent à cette totalité leur sens invisible en dehors de cette totalité ». E. Lévinas, Totalité et Infini. Essai sur l'extériorité, Paris, LGF, Le Livre de poche, Coll. Biblio-essais, 1990, p. 6.

13. L. Lavelle, De l'Être, op. cit., p. 48.

14. Pour Lévinas, l'utilisation du terme de «vie» dénonce bien souvent un discours situé après la contractualisation de l'existant et de l'existence. Parler de « vie » c'est prendre pour acquis ce rapport plutôt que de s'y intéresser à son niveau ontologique et métaphysique. Cf. De l'existence à l'existant, Paris, $3^{\mathrm{ème}}$ éd., 1993, p. 29.

15. Ibid., p. 12. 
étant, dont le mal premier n'est pas l'insatisfaction subjective de vivre ou d'achever ses ambitions mais l'impossibilité de fuite hors d'une présence objective. Pour le dire selon les termes de De l'évasion, l'insatisfaction reconnue par Lavelle n'est qu'une variation plus ou moins romantique d'un problème plus profond et plus insoluble, dans la mesure où ces désespoirs existentiels «ne remettent pas en cause l'être, et obéissent à un besoin de transcender les limites de l'être fini $»^{16}$. Ce n'est pas le choix qui exalte ou désespère l'individu qu'exprime le besoin d'évasion lévinassien mais le refus de l'être ou de la présence.

Nous disposons donc de ce «sentiment irréductible qu'il y a de l'être $»^{17}$, et du fait que le moi est pris dans un tout plus vaste qui le précède, le soutient, lui succède, bref, demeure indéfectiblement là. Cette conception de l'être nous éloigne alors d'une vision substantialiste de ce dernier. Si Lavelle conçoit l'être comme une activité, une source infinie de création, qui se recrée sans cesse, pour Lévinas, cette recherche de l'être pur nous mène vers un presque rien, vers une absence universelle d'étants, où il ne demeure pas moins une présence résiduelle et inévitable : celle d'un quelque chose ou plutôt du fait qu'il y a quelque chose. Nous tombons sur un «être anonyme qu'aucun étant ne revendique, être sans étants ou sans êtres ${ }^{18}$. Par ses propos, Lévinas nous semble donc radicaliser l'expérience de pensée lavellienne, poussant l'imagination jusqu'au souci d'atteindre l'être pur et séparé de ce qu'il produit. Cette existence désertique s'éloigne de l'être lavellien conçu comme tout concret de ce qui est, étrangement comparé par Lavelle à un visage : l'ensemble des choses comprises dans l'être sont comme les aspects d'un visage qui ne tirent leur réalité qu'au sein même de ce visage qu'elles se chargent d'exprimer et de manifester, sans que lui-même se manifeste dans l'apparition de sa délimitation. Mais si la conception de l'être diverge, en ce que Lavelle y voit une source chaleureuse de déterminations, alors que Lévinas y voit plutôt le désert froid de l'indéterminé, ils s'accordent pourtant pour dire de l'être qu'il n'est ni une qualité, ni un genre auquel l'individu appartient, mais ce que tout étant présuppose. Chez l'un comme chez l'autre, nous retrouvons donc l'idée que l'être est l'apriori universel de mon existence. L'être est renvoyé à la source de l'activité, présence qui fait être, Lévinas nous disant qu'il est « le fait par lequel il y a tout ce qu'il y a » ${ }^{19}$ ou Lavelle que : «l'être est précisément le caractère qui fait que les choses sont des choses $»^{20}$. Pour autant, la reconduction de l'être à sa verbalité se traduit non pas par la caractérisation temporelle de ce verbe mais bien au contraire par son caractère définitif et infinitif, puisque Lavelle comme Lévinas posent l'être en son éternité. Cette équation se manifeste par l'impossibilité affirmée de trouver un

16. E. Lévinas, De l'évasion, op. cit., p. 96.

17. Id. « Recension de L. Lavelle, La Présence totale », op. cit., p. 393.

18. Id. De l'existence à l'existant, op. cit., préface à la deuxième édition.

19. Ibid., p. 23.

20. L. Lavelle, op. cit., p. 44. 
commencement à l'être quand il s'agit d'accuser l'impossibilité de faire sortir l'être du néant, Lavelle affirmant qu' «il ne peut pas y avoir de naissance de l'être ${ }^{21}$ tandis que Lévinas insiste sur cette impossibilité de « concevoir le commencement de l'être »". Pour Lavelle, puis pour Lévinas, l'existence pure est aussi le nom de l'éternité, où l'on ne peut trouver d'individus extérieurs les uns aux autres, indépendants de l'être et en relation avec lui. Il s'agit d'une présence atemporelle hissée au rang de fait ontologique antérieur à toute représentation par une conscience.

\section{La subjectivation de l'être}

Contre l'idéalisme subjectiviste posant que la pensée du sujet fonde sa propre existence et l'existence du réel, Lavelle se montre soucieux de faire précéder la pensée par l'existence, comme en témoigne sa lecture de la deuxième Méditation métaphysique de Descartes : le cogito est certes l'appréhension de son existence par un sujet mais cette découverte, première chronologiquement, ne l'est pas ontologiquement. L'existence en général n'est pas fondée par cette découverte et il ne s'agit, dans cette expérience de pensée, que de la découverte de l'existence en tant que telle ${ }^{23}$. Mais s'il s'oppose au subjectivisme, Lavelle n'entend pas moins proposer une philosophie du sujet, plus juste, qui restituera sa genèse ${ }^{24}$ au sein de l'être. Proposition retenue par Lévinas qui, dès De l'existence à l'existant jusqu'à Totalité et Infini, s'évertue à décrire la formation du sujet dans son rapport distinctif à l'être. Être pour un sujet consiste donc pour Lavelle dans le fait d'une adhésion: "le moi ne peut subsister et s'accroître que par une adhérence à l'être ${ }^{25}$. Définition reprise par Lévinas qui en viendra à comprendre le sujet comme adhérence à l'être ${ }^{26}$. Chez nos deux auteurs, l'ontologie devient donc identifiable par une structure identitaire ou tautologique, revenant à dire que l'être adhère à lui-même ou qu'être personnellement c'est adhérer à l'être. Loin que cette tautologie n'appauvrisse l'existence, elle en révèle l'essence, comme le remarque Lavelle :

\section{Ibid., p. 41.}

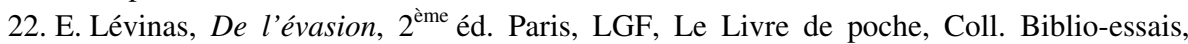
1998, p. 121.

23. «Les esprits les moins prévenus sont prêts à reconnaître que si, en disant "je pense", Descartes appréhende du même coup sa propre existence, cela ne signifie pas que l'existence en général soit postérieure à la pensée et fondée par elle, mais seulement que c'est dans sa propre pensée qu'il saisit indubitablement la première révélation de l'existence ». L. Lavelle, op. cit., p. 198.

24. Ibid., p. 16.

25. Ibid., p. 248.

26. E. Lévinas, De l'existence à l'existant, op. cit., p. 28. 
Et il est remarquable que le jugement «l'être est », par lequel s'exprime avec le plus de hardiesse l'ambition ontologique de la pensée, semble à la fois résumer dans la perfection de sa tautologie l'essence de tous les jugements d'inhérence et fournir à ceuxci un cadre immuable qu'il leur appartient de remplir ${ }^{27}$.

\section{ou le principe qui est l'identité elle-même pour Lévinas :}

L'être est: il n'y a rien à ajouter à cette affirmation tant que l'on n'envisage dans un être que son existence. Cette référence à soi-même, c'est précisément ce que l'on dit quand on parle de l'identité de l'être. L'identité n'est pas une propriété de l'être et ne saurait consister en une ressemblance de propriétés qui supposent ellesmêmes l'identitée ${ }^{28}$.

Pour Lavelle, cette antériorité de l'être sur nous-mêmes éprouve notre prétention à être, appelant la conversion de cette ambition en opération par laquelle il nous faut réaliser la possibilité d'être que nous sommes. Hypothèse que Lévinas semble bien prolonger en employant le terme d'inversion ${ }^{29}$ par laquelle l'être devient l'être d'un étant ou encore $\mathrm{d}^{\prime}$ hypostase $\mathrm{e}^{30}$. Pour nos deux auteurs, être un sujet signifie essentiellement être indépendant de l'être et néanmoins inscrit en lui. Je ne me dispose à cette indépendance qu'à proportion que je fais mien cet acte pur ou cette activité d'exister. Vis à vis de l'être, encore appelé absolu, acte pur, Tout, ou présence totale, la métaphysique lavellienne propose alors une variation de termes correspondants pour désigner le sujet, qualifié tantôt de possibilité, de partie, ou de puissance de l'être. La relation de constitution du sujet est actualisation de la possibilité que nous sommes par une participation à ce qui nous fait être ${ }^{31}$. Lavelle s'éloigne du sens platonicien en indiquant que si le moi prend part à l'être-source, il y va non pas d'une imitation mais d'une initiative afin de s'approprier l'acte auquel il participe. De même, Lévinas estime que «dans le fait d'exister (...) s'accomplit un évènement non pareil et préalable de participation à l'existence, un évènement de naissance ${ }^{32}$. Il récuse le sens platonicien du terme dans la mesure où la participation n'est pas entrée en relation de deux termes subsistants mais émergence d'un étant qui adhère, en l'assumant, à ce qui existe déjà, au risque de s'y

27. Ibid., p. 153.

28. E. Lévinas, De l'évasion, op. cit., p. 93.

29. «l'être en général ne devient-il pas l'être d'un "étant" par une inversion (...) ?» Ibid., p. 18.

30. « dans l'être impersonnel surgit, comme par l'effet d'une hypostase, un être, un sujet, un existant »Idem.

31. «Toutefois, s'il y a une expérience reconnue et pour ainsi dire populaire de la participation, si le mot même a un sens et désigne une sortie de soi par laquelle chacun éprouve la présence d'une réalité qui le dé-passe, mais qu'il est capable de faire sienne, cette participation, qui est toujours active ou affective, et active jusque dans l'affection qu'elle produit, est aussi une coopération, c'est-à-dire non point une opération répétée et imitée, mais une action commune, et dont chacun prend sa part ou qu'il consent à assumer ». L. Lavelle, De l'Être, op. cit., p. 19.

32. E. Lévinas, De l'existence à l'existant, op. cit., p. 26. 
confondre $^{33}$. Lavelle donne un sens inédit au terme de participation, puisque la découverte de notre existence et donc de notre inscription dans l'être revient d'ores et déjà à participer. La conscience, lorsqu'elle s'aperçoit pour la première fois qu'elle n'est pas le tout de l'être - mais qu'elle en est - en vient à se saisir comme puissance créatrice - empruntée à celle de l'être. Si participer à l'être revient pour Lavelle à actualiser des possibles, il s'agit pour Lévinas d'investir cette existence qui se fait sans moi comme mon existence à faire ${ }^{34}$. Chez ce dernier, l'acte consiste en un dédoublement de l'existence par lequel celle-ci se perd et se récupère. Par ce dédoublement, l'être se scinde comme ce qui est et ce qui se possède, ce qui est et ce qui $\grave{a}$ être ou à faire. Cette possession de l'existence en un sujet est d'abord une « source de souci, avant d'être source de jouissance ${ }^{35}$ comme il l'affirmera dans Totalité et Infini.

Cet acte de participation est pour Lavelle comme pour Lévinas un acte de prise de conscience de soi $^{36}$ par laquelle le sujet s'approprie l'existence ${ }^{37}$ : activité par laquelle s'opère un retour à soi et une possession de soi, que Lavelle $^{38}$ désigne par les termes d'intériorité et d'intimité, tout comme Lévinas $^{39}$. Être personnellement, c'est donc aller vers ce qui fait être afin de s'approprier cet acte devenant en nous être à faire, une activité de façonner son existence. Cette participation est donc appropriation de cette production constante de l'exister et délimitation d'un moi existant. Autrement dit, pour Lavelle et Lévinas, le sujet, en étant conscient de soi, crée une dualité

33. Dans De l'existence à l'existant, op. cit., p. 98-99, Lévinas précise son concept de participation en se positionnant par rapport à Platon et Lévy-Bruhl. Comme ce dernier, il s'éloigne du sens platonicien selon lequel participer c'est prendre part à l'essence d'un concept. Il voit dans la participation mystique décrite par Lévy-Bruhl, une similitude avec l'il $y a$, puisque participer ne fait pas coexister un terme avec un attribut mais lui fait devenir ce à quoi il participe. Les termes en jeu perdent alors leur substantivité et retournent à un fond indistinct. La participation mystique implique la possession intime d'un terme par l'autre et «le sujet est à la fois lui-même et l'être dont il participe»-cité par Benjamin Fondane, «Lévy-Bruhl et la métaphysique de la connaissance », Revue philosophique de la France et de l'étranger, mai-juin, 1940, volume CXXIX, p. 29-30.

34. Lévinas appelle une "conquête de l'être », mais aussi régulièrement un accueil et un positionnement. Cf. De l'existence à l'existant, op. cit., p. 26.

35. Ibid., p. 36.

36. Par le positionnement, la conscience peut émerger. La conscience est d'abord définie par Lévinas comme possible évasion de l'il y a dans le sommeil notamment, mais elle est aussi évènement réflexif : elle est synonyme de recul, ce qui signifie concrètement qu'elle peut reprendre ce qui la précède. À un niveau ontologique, elle est perte et récupération de l'existence. Cf. Ibid., p. 116 et 124.

37. Pour Lévinas, l'émergence d'un sujet et de l'existence impersonnelle surgit non comme une relation mais comme une participation, c'est-à-dire comme un dédoublement minimal par quoi nous nous apercevons de l'être dans lequel nous sommes déjà, voire par quoi nous nous en étonnons. L'expérience du dédoublement part d'une expérience de l'étrangeté de l'être qui est une « autre manière de l'assumer». Ibid., p. 28.

38. «L'être ne peut donc être perçu que comme l'intimité totale ou comme un moi universel ». L. Lavelle, De l'Être, op. cit., p. 134.

39. «Le moi possède un soi (...) relation qu'on appelle intimité ». E. Lévinas, De l'existence à l'existant, op. cit., p. 37-38. 
entre l'être et lui-même et délimite sa subjectivité dans le tout de l'être. En des termes lavelliens, il s'agit de participer à l'acte pur qui soutient son existence et qui l'appelle à actualiser la puissance qu'il est ${ }^{40}$. En des termes lévinassiens, nous dirons qu' exister personnellement c'est commencer et agir puisque «l'acte est l'inscription même dans l'être » ${ }^{41}$. Accord fondamental entre ces deux penseurs, puisque Lavelle considère l'identité de l'être et de l'acte comme la «clé de la métaphysique ${ }^{42}$ permettant de comprendre qu'être c'est avant tout agir, ou plus précisément que l'être-acte incite à l'acte d'être. Cet acte d'être s'éprouve alors pour Lévinas comme une conquête permanente de ce qui le précède, proposition qui fait singulièrement écho aux propos de Lavelle :

Car qui ne vérifie à chaque instant au fond de lui-même la formule traditionnelle, qu'être, c'est agir ? où le moi dispose d'une puissance omniprésente et qui le dépasse, mais qui se change nécessairement en action dès qu'elle rencontre une borne qu'elle essaie de vaincre, mais qui la détermine et lui donne, pour ainsi dire, un objet ou un contenu ${ }^{43}$ ?

Lévinas partage bien l'idée qu'être, pour un moi, c'est agir et commencer à agir. Le retour phénoménologique au vécu de lassitude lui permet de relever un refus de jouer le jeu de l'existence, comme il sert de révélateur de la dualité de l'être et de l'étant. Dans le vécu de lassitude, l'existence apparaît comme «appel d'un engagement à exister » ${ }^{44}$ auquel le sujet hésite à répondre. Lavelle, et à sa suite Lévinas, posent donc que le sujet n'existe que par une initiative, soit que celle-ci reprenne à son compte celle de l'acte pur (Lavelle), soit qu'elle prenne la forme d'un effort pour être (Lévinas). La métaphysique lavellienne considère donc que notre accomplissement passe par deux étapes : retrouver en nous le principe de création du tout - l'acte pur - et ensuite s'efforcer de l'actualiser dès que faire se peut. La personnalité étant à la fois l'expression de l'être et un aspect de l'être différents de tous les autres. Ceci revient aussi à dire que l'on ne peut distinguer un sujet dans le Tout qu'en lui attribuant certaines qualités qui le distinguent. Tout se passe comme si la personnalisation était à la fois un effort de détermination et de délimitation des caractères, conjugué à un effort d'actualisation de la plus grande diversité de virtualités de l'être auquel nous appartenons. La tentation inverse à cet effort de personnalisation étant la confusion avec le tout donné, au lieu de retrouver le tout donnant: "l'union avec l'être pur réside tout entière dans une opération qui fonde sa personnalité, au lieu de la laisser se dissoudre et

40. Cette participation peut s'effectuer non seulement par la prise de conscience de l'être qui nous dépasse, mais aussi par l'œuvre indéfinie de connaissance de cette totalité, ou encore par la volonté d'agir qui contribue à réaliser notre essence.

41. E. Lévinas, De l'existence à l'existant, op. cit., p. 37.

42. L. Lavelle, De l'Être, op. cit., p. 301.

43. Ibid., p. 293.

44. E. Lévinas, De l'existence à l'existant, op. cit., p. 31. 
s'anéantir dans le tout ${ }^{45}$. La philosophie lévinassienne, qualifiée de pensée de la séparation, ne peut alors que souscrire à cette idée en la radicalisant. Il s'agit en effet pour Lévinas de signaler le péril de l'absolution dans l'être anonyme ou l'il y a, fond indistinct. Toutefois, la distinction subjective ne provient pas pour lui d'une telle élaboration dialectique ; elle ne passe pas par la caractérisation de la partie par rapport au tout mais par une autopossession de soi et surtout, par l'assignation d'autrui à ma responsabilité de sujet $^{46}$. Lévinas tendant à radicaliser l'évasion et la formation individuelle d'un étant, ne fait pas de cette opération un processus de détermination qualitative mais phénoménologique : il s'agit de la différence de vécu ${ }^{47}$.

Par-delà ces différences, la «phénoménologie dialectique ${ }^{48}$ de Louis Lavelle rencontre pourtant encore la pensée lévinassienne, quand elle se propose de penser cette distinction du sujet avec l'être grâce à la notion d'instant. Pour Lavelle, l'accomplissement de la possibilité que nous sommes se joue à chaque instant de notre existence ${ }^{49}$. Tout instant représente alors une offre de participation qui permettra le passage du possible à l'actualité, et dans laquelle s'introduit l'initiative personnelle et le mérite consécutif de cet effort. Ainsi Lavelle écrit-il que : "chaque conscience se heurte en tout instant à sa propre limitation, mais qu'en tout instant elle doit faire effort pour la surmonter $»^{50}$. C'est cette proposition que nous paraît retenir Lévinas pour qui l'émergence du sujet ne se comprend que par une dialectique de l'instant ${ }^{51}$; c'est-à-dire par un effort pour saisir cet atome temporel, grâce auquel nous nous constituons et nous posons dans l'être. Lavelle semble très proche de Lévinas lorsqu'il écrit :

(...) possibilité pure et simple, permission d'agir ; c'est l'instant qui transforme cette possibilité en être, qui décide du oui ou non. Et le temps tout entier peut-être défini comme le rythme des oui et des non. Il dessine, comme l'avait vu Bergson, la forme de notre hésitation. En lui la vie est sans cesse essayée, refusée et reprise ${ }^{52}$.

45. L. Lavelle, De l'Être, op. cit., p. 49.

46. «Être moi signifie dès lors ne pas pouvoir se dérober à la responsabilité ». E. Lévinas, En découvrant l'existence avec Husserl et Heidegger, Paris, Vrin, 2010, p. 273.

47. Ce vécu se joue dans la prise conscience de soi, dans cet effort pour assumer ce qui existe déjà mais aussi par l'inscription physique dans le monde grâce auxquels le sujet constitue une intériorité conçue comme égoïsme.

48. Expression d'Hervé Barreau dans sa conférence «L'apport de Bergson à Lavelle», Bulletin de l'association Lavelle, bulletin $\mathrm{n}^{\circ} 22$, septembre 2011.

49. «Quant à ce sujet lui-même, il réside dans l'acte intérieur qu'il accomplit et que l'on ne peut pas réduire à la pensée d'un objet ou d'une chose, car le propre de cet acte, c'est d'engager l'existence même du moi, c'est de la faire être dans une opération qu'il lui faut accomplir (...) qui fait de lui à chaque instant le premier commencement de lui-même (...)». L. Lavelle, De l'Être, op. cit., p. 16-17.

50. Id. La Présence totale, op. cit., p. 16.

51. «fouiller l'instant, en chercher la dialectique (...)». E. Lévinas, De l'existence à l'existant, op. cit., p. 42.

52. L. Lavelle, «Le rythme du temps », Le Temps, 7 février 1937, repris in Science, esthétique, métaphysique, Paris, Albin Michel, 1967, p. 204. 
L'instant est donc assimilé à la faculté d'affirmer ou de refuser, d'entrer dans l'être ou de s'y abandonner passivement, il est la possibilité des contraires, l'hésitation constitutive de notre liberté. Pour Lévinas, c'est dans l'hésitation à s'emparer de l'existence - comme dans la paresse, la lassitude ou la fatigue - que l'instant apparaît comme ce qui médiatise la relation de l'existant à l'existence ${ }^{53}$. D'ailleurs, Lévinas note dans sa recension de La Présence totale que la pensée de Lavelle permet un dépassement du pessimisme allemand, comme elle donne au sujet la maîtrise de son destin dans la conquête du présent : «elle nous invite à accomplir l'effort constant pour reconquérir le présent au lieu de le fuir ${ }^{54}$. Lévinas sera donc très sensible à cette idée, exposant dans ses premières œuvres, la genèse d'un sujet s'efforçant à exister, se demandant : " si l'instant n'est pas l'évènement même par lequel dans le pur acte, dans le pur verbe d'être, dans l'être en général, se pose un étant ${ }^{55}$. Le sujet ne décide pas alors d'exister, il assume ce qui existe déjà. En d'autres termes, avoir conscience d'une existence que l'on fait sienne, c'est être présent à soi, et cette présence se fait dans la saisie de l'instant. Grâce à la saisie d'un instant présent, un sujet se présente. L'instant a pour fonction ontologique d'inscrire la position du sujet dans le tout de l'être : l'instant se réfère uniquement à soi et accomplit l'hypostase. Dans l'acte de commencer, parfois refusé par le sujet lassé, l'instant doit être lâché et récupéré et c'est ainsi que l'existence se dédouble et se possède en moi. Dans l'instant, l'existence se rapporte à elle-même d'une façon originale; cette originalité forme un sujet conscient ${ }^{56}$.

Mais si l'instant permet la naissance du sujet à même l'existence, c'est parce que l'être est justement atemporel ou éternel. Par le temps, nous sommes divisés avec nous-mêmes - nos vécus se succèdent - et surtout divisés avec l'être. Ainsi que l'écrit Lavelle : «Le sujet divise et échelonne la présence, (...) il la filtre à travers le guichet de l'instant ${ }^{57}$. Par l'instant, l'être est divisé. C'est dans cette division, succession - voire éclatement que des sujets peuvent apparaître et se distinguer. De même pour Lévinas, si l'être est «sans interruption possible ${ }^{58}$, nous comprenons mieux pourquoi là encore, l'instant permet de diviser l'être. Perçant la trame de l'éternité, divisant ce qui n'était pas divisible à partir d'un instant, un sujet peut commencer. Le sujet ne peut réellement se distinguer avec l'être éternel et

53. La dualité n'est pas manifeste dans la relation entre des termes subsistants par soi et entrant en relation mais dans ces vécus où l'assomption de l'existence est manifeste par sa difficulté. La dualité des termes apparaît dans l'échec pour un étant d'adhérer à l'existence.

54. E. Lévinas, « Recension de L. Lavelle, La Présence totale », Recherches Philosophiques, op. cit., p. 394.

55. Id. De l'existence à l'existant, op. cit., p. 16.

56. La conscience est donc bien un recul par quoi l'existence apparaît pour elle-même et se possède. Si l'instant articule cette déprise et cette reprise de l'existence, Lévinas donne alors une définition temporelle de la conscience : elle est instant qui se récupère.

57. L. Lavelle, La Présence totale, op. cit., p. 162.

58. E. Lévinas, Autrement qu'être ou au-delà de l'essence, $2^{\text {ème }}$ éd. Paris, LGF, Le Livre de poche, Coll. Biblio-essais, 1990, p. 47. 
anonyme que par sa temporalité constitutive d'occasions, d'actions, de démarches personnelles, de conscience de soi. Un étant sans temps ne bénéficierait pas de l'écart constitutif d'une conscience, grâce à laquelle il saurait qu'il se rapporte à l'être et qu'il en fait partie. De Lavelle à Lévinas, nous constatons une même définition de la conscience, caractérisée par le fait qu'elle advient en se saisissant de cette charge d'être, dans le jaillissement de l'instant, brisant ainsi la continuité ignorante d'elle-même.

\section{L'instant lévinassien contre l'instant lavellien}

Si nous avons entrepris de démontrer la filiation métaphysique entre Emmanuel Lévinas et Louis Lavelle, il semble pourtant que l'enthousiasme de notre auteur doive trouver sa limite là même où il trouvait son ouverture :

Le grand mérite de M. Lavelle consiste à nous rendre sensible cette vérité que la réhabilitation du présent est le seul moyen de briser le jeu tragique du temps. Mais chez M. Lavelle la victoire sur le temps est une sortie en dehors du temps. Bien que l'être qui supporte le sujet soit un acte pur et nullement une substance, le présent de M. Lavelle est intemporel. C'est le carrefour du temps et de l'éternité. L'acte pur est pour M. Lavelle, au sens aristotélicien, accomplissement, achèvement et joie plus qu'effort ${ }^{59}$.

En somme, Lévinas félicite Lavelle d'avoir édifié une structure temporelle par laquelle s'engage un salut du sujet hors du pessimisme heideggérien, le pensant comme jeté dans l'existence et voué à la mort. La réhabilitation de l'instant présent ${ }^{60}$, comme lieu de constitution de soi et d'engagement à être, est saluée et reprise par la genèse lévinassienne. Le présent n'étant plus relativisé et confondu dans une série temporelle homogène, il est ce qui fonde la participation à l'être. Mais, ce que déplore Lévinas, c'est justement que ce dépassement du pessimisme allemand ne soit en réalité qu'une façon de se soumettre à l'être éternel. Cette soumission se révèle dans la demi-réhabilitation du présent, qui n'est pas vraiment un instant concret du temps mais une éternité concrétisée chez Lavelle. Pour Lavelle, le temps est une phénoménalisation de l'être d'une façon successive ; le moyen de sa manifestation aux yeux d'une conscience finie, ne pouvant que diviser l'être en quantité d'aspects à considérer successivement. Comme le dit R. Schönberger dans un article, Lavelle en liant l'être et la présence a exprimé «ce qui était pour Heidegger une implication irréfléchie de la tradition classique; l'être, présumé hors du temps, parvient à l'expression grâce à une détermination temporelle (la

59. Id. « Recension de L. Lavelle, La Pésence totale», op. cit., p. 395.

60. Notons qu'une partie du chapitre II de De l'Être, s'intitule «Privilège ontologique de l'instant ». 
présence, l'actualité) $»^{61}$. Au fond, Lavelle propose une définition métaphysique du présent: l'être est présence et le présent signifie la constance à être. L'instant est la spécification dynamique de la présence : il offre la prise de contact avec la présence totale et éternelle, assimilée à Dieu. Ainsi, le sujet participe à la présence à chaque instant, de sorte que l'instant réalise la croisée du temps et de l'éternité. L'instant lavellien est une offre d'actualisation de l'être par lequel ce dernier se manifeste comme événement unique, riche de possibilités à actualiser ${ }^{62}$. Si l'être éternel peut laisser s'inscrire en lui une existence temporelle c'est justement, parce que l'instant est à la fois un fragment d'absolu et ce qui permet de le diviser selon la forme d'une succession. Finalement, la dynamique de l'existence temporelle permet de contacter l'être lui-même dynamique, conçu comme une activité. C'est bien l'inverse de la conception lévinassienne pour laquelle l'instant est ce moment permettant de garantir la distinction d'avec l'être continu et éternel ${ }^{63}$. Pour lui, l'instant doit offrir un renouveau et permettre au « je » de s'arracher à l'irrémissible présence de l'être plutôt que de favoriser « une rencontre du temps et de l'absolu ${ }^{64}$. Ce sujet ou cet instant dont il est porteur est exigence d'un recommencement. Ainsi Lévinas écrit: «La "personnalité" de l'être est son besoin même du temps comme d'une fécondité miraculeuse dans l'instant lui-même par lequel il recommence comme autre $»^{65}$.

Lévinas reconnaît que cette déception est bien le fruit d'une attente philosophique personnelle, et qu'il ne peut reprocher à Lavelle d'avoir manqué de penser ce que ce dernier n'entendait pas viser. S'il est donc «ridicule de reprocher à une pensée rigoureuse et procédant d'une inspiration authentique de ne pas avoir soutenu ce qu'elle n'a pas eu l'intention de soutenir ${ }^{66}$, Lévinas remarque qu'une pensée vraie se trouve toujours excédée par les horizons qu'elle ouvre et qu'elle appelle implicitement. Dans ces dernières lignes de la recension de La Présence totale, nous pouvons sentir que Lévinas a d'ores et déjà regardé dans leur direction, puisqu'il se montrera soucieux de reprendre cette réhabilitation du présent, en en faisant non pas une éternité présente mais un présent éternel. Plutôt que d'opposer l'éternité au temps, il s'agit pour Lévinas de

61. R. Schönberger, «Louis Lavelle: l'expérience de l'être comme acte», Archives de philosophie, $\mathrm{n}^{\circ} 53,1990$, p. 279.

62. L'événement perçu est le présent, l'événement oublié est un souvenir qui peut s'actualiser, l'événement anticipé est en attente d'actualisation. Présent, passé et futur sont des modalités de représentation et de rapport à l'événement produit dans l'instant. Le passage de l'événement perçu à l'événement anticipé ou remémoré est alors ce que Lavelle appelle le temps.

63. «Mais l'on peut se demander si l'évanescence du présent n'est pas la seule possibilité pour un sujet de surgir dans l'être anonyme et d'être susceptible de temps ». E. Lévinas, $D e$ l'existence à l'existant, op. cit., p. 124.

64. Ibid., p. 170.

65. Ibid., p. 159.

66. E. Lévinas, « Recension de L. Lavelle, La présence totale », op. cit., p. 395. 
comprendre que s'opposent en réalité deux présents. Si l'éternité est ce en quoi tout est tout entier présent, le temps est ce en quoi le présent rompt avec un passé et se distingue d'un à-venir. Ces deux présents sont dérivés de deux formes de présence : la présence de l'être est sans distance, la présence de l'étant exprime une distance d'avec l'être. La présence de l'être est éternelle et désincarnée, tandis que le présent est temporel parce que concret et changeant. En somme, commencer, c'est assumer l'existence comme sienne à chaque instant, dans un effort et donc par l'œuvre de son corps s'efforçant, alors que chez Lavelle, la participation est finalement collaboration à l'acte pur, achèvement d'une pensée consciente d'elle-même et de l'être, se possédant avec joie. Nous voyons donc bien la différence qui sépare Lévinas de Lavelle puisqu'il ne peut y avoir de temporalisation sans cette différenciation concrète, éprouvée avec l'éternité. Cette différence sera d'autant plus profonde que chez Lévinas, la saisie de l'instant - tranchant sur l'éternité - sera elle-même insuffisante pour constituer un temps véritable. Dès le troisième chapitre de De l'existence à l'existant, il insistera sur l'idée que l'instant, s'il permet une démarcation personnelle avec l'être, demeure le moyen d'une identification et d'une référence du sujet à lui-même par lesquelles il s'enchaîne à soi. Or il n'est pas certain que l'identification et la subjectivation par l'instant soient garantes d'une radicale sortie de l'être. De même, cette succession d'instants ne constitue pas encore une temporalité conçue comme renouvellement. Ce qui constituera la temporalité véritable, c'est non seulement de briser la présence indistincte par l'instant, mais surtout de la diviser en des moments tout à fait autres. Or cette altérité de l'avenir ne peut être donnée par l'instant seul mais par le surgissement de l'autre, garant d'une nouveauté véritable ${ }^{67}$.

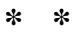

$*$

La philosophie d'Emmanuel Lévinas tente de penser la naissance de la subjectivité non pas à partir du rien ou de lui-même, mais de cet être éternel où sa naissance le plonge. Cette intuition initiale d'une inscription dans une totalité plus vaste et dont il faut se distinguer n'est pourtant pas absolument originale et trouve notamment ses racines dans la philosophie de Louis Lavelle, ainsi que la recension de 1934 nous l'indique explicitement. Nous avons observé une parenté philosophique, fondée sur l'identification de l'être à un acte, mais aussi sur l'inscription du sujet dans l'être et sa distinction avec lui. De même, la naissance de la subjectivité renvoie chez nos deux auteurs à un instant que le sujet saisit pour briser la continuité éternelle de l'être et par lequel il y participe à sa façon.

67. «L'altérité absolue de l'autre instant (...) ne peut se trouver dans le sujet qui est définitivement lui-même. Celle altérité ne me vient que d'autrui ». Id., De l'existence à l'existant, op. cit., p. 160. 
Nous noterons pourtant la profonde divergence de vue entre une pensée chrétienne du désir pour l'être-source, et une pensée juive de la séparation radicale avec Dieu. En outre, Lévinas déplore la semi-réhabilitation du présent, manifestant son souci de comprendre le temps comme ce qui peut permettre d'exister personnellement et concrètement. Loin de produire une durée authentique, conçue distinctement de l'éternité, l'instant lavellien est «un carrefour du temps et de l'éternité » ${ }^{68}$ reprenant à sa façon la soumission du temps et du sujet à l'être éternel. Lévinas, quant à lui, ne considère pas l'instant comme le résidu d'une présence éternelle mais comme ce à partir de quoi l'individu se positionne et se distingue de l'être. Il estimera pourtant, dès 1941, que l'instant n'est pas un gage suffisant d'altérité et de temporalité. 